EGU2020-18412

https://doi.org/10.5194/egusphere-egu2020-18412

EGU General Assembly 2020

(c) Author(s) 2021. This work is distributed under

the Creative Commons Attribution 4.0 License.

\title{
Flow regimes, grain mobility and size segregation in stationary bi- disperse granular flows
}

\author{
Tomas Trewhela ${ }^{1}$, Nico Gray ${ }^{2}$, and Christophe Ancey ${ }^{1}$ \\ 'École Polytechnique Fédérale de Lausanne, Environmental Hydraulics Laboratory, ENAC, Lausanne, Switzerland. \\ ${ }^{2}$ Department of Mathematics and Manchester Centre for Nonlinear Dynamics, The University of Manchester, Manchester, \\ United Kingdom.
}

We studied granular flows of glass beads on an inclined conveyor channel. An upward-moving belt conveyed particles that flowed down the channel under the action of gravity, thus creating a stationary flow. To visualize the internal dynamics of the bulk, we relied on the refractive index matching technique. Under fixed slope and belt velocity, we ran mono- and bi-disperse experiments to characterize spatially and temporally the dynamics and concentration fields of these granular flows. Mono-disperse experiments were done using 6 and $8 \mathrm{~mm}$ beads on slopes of $10,12,15$ and $18^{\circ}$ and 3 different belt velocities. Beads of $14 \mathrm{~mm}$ were added in concentrations of $10,20,30$ and $40 \%$ for the bi-disperse experiments. The rear part of the flow exhibited wellarranged particle layers that moved relatively between them. This particle arrangement ended with a sharp transition to the front of the flow and a dilated convective front. Bi-disperse experiments with low concentrations of large particles conserved the same layered-convective regime with the few added large beads confined to the convective front, a result of size segregation. When the concentration of large beads was increased to 30\%, the described regime disappeared. Large grains were frequently dragged back by the belt, thus disrupting the arrangement of particle layers. A quasi-stationary behavior was observed in these experiments, small particles migrated to the front of the flow in pulses that after a while were dragged back, repeating the cycle. We observed that particle concentration fields, on average, were consistent with the structures observed for the breaking size-segregation wave phenomenon. The effective basal friction, local concentrations and dilation, among other variables, are responsible for these phenomena. 